\title{
Information und Informationsverarbeitung
}

\section{Teil 2: Das Internet und die Pflege emotionaler Intelligenz}

\author{
Bernd Markert $\cdot$ Stefan Fraenzle $\cdot$ Christoph Tieben
}

Erhalten: 23. Juni 2009/Akzeptiert: 28. Juni 2009/Online veröffentlicht: 11. September 2009

(C) Springer-Verlag 2009

\section{Einleitung}

Nachdem unser letzter Kommentar zur Information und Informationsverarbeitung (Markert und Fraenzle 2007) aufgrund der notwendigen Kürze nicht alle uns wichtig erscheinenden Details berücksichtigen konnte, sind wir der Redaktion der UWSF dankbar, uns einen zweiten Teil mit einigen neueren und neuesten Entwicklungen der Informationsverarbeitung zu eröffnen.

Das menschliche Gehirn kann als zentraler Integrationsund Verarbeitungsort aller Informationen betrachtet werden (Radio Bremen 2006; Roth 2003; Spitzer 2007). Allerdings dringt nicht jede Information bis zur Hirnrinde vor, also in das Bewusstsein hinein, sondern nur ein winziger Bruchteil davon. Sonst würden wir weder zeitnah handeln können noch geistig gesund bleiben. Eine Vielzahl von Zentren dienen der unbewussten Vorverarbeitung von Signalen. Diese häufig in Reflexbögen ablaufenden Reaktionen werden auch

Die Onlineversion dieses zweiteiligen Beitrages enthält Teil 1 (Markert und Fränzle 2007) mit dem Titel: „Die Stufen der Erkenntnis und das Verhältnis von Objektivität und Subjektivität" (doi: 10.1065/ uwsf2007.3.173)

Dieser Kommentar ist Joseph Weizenbaum gewidmet, der am 8. März 2008 verstarb. Die Möglichkeit, mit seinem elektronischen Psychiater ELIZA (auch auf Deutsch) zu kommunizieren, wird im Text eröffnet.

Bernd Markert · Stefan Fraenzle

Internationales Hochschulinstitut Zittau,

Lehrstuhl für Umweltverfahrenstechnik,

Markt 23, 02763 Zittau, Deutschland

Bernd Markert $(\square)$

Fliederweg 17, 49733 Haren/Erika, Deutschland

E-Mail: markert@schlundmail.de

Christoph Tieben

Nordstrasse 50a, 49733 Haren, Deutschland als autonomes Nervensystem bezeichnet und dienen der Koordination vegetativer Funktionsabläufe wie Atmung, Verdauung und Kreislauffunktion. Durch das „Nicht-Bewusstmachen" einer Vielzahl alltäglicher und sich ständig wiederholender Prozesse werden diese beschleunigt, da sie nicht einer ständigen bewussten Kontrolle unterliegen.

Obwohl in den vergangenen Jahrzehnten eine enorme Entwicklung in der Erforschung einzelner Gehirn- und Nervenprozesse sowohl morphologischer als auch physiologischer Natur in der Medizin und in verwandten Naturwissenschaften stattgefunden hat, ist derzeit aufgrund der Komplexität noch relativ wenig über die Gehirnleistung und deren exakten Steuerung bekannt. Dies betrifft insbesondere auch Fragen zur Leistungsfähigkeit. Obwohl das menschliche Gehirn nur 2\% der Körpermasse ausmacht, müssen dennoch etwa $20 \%$ des Blutes vom Herzen ins Gehirn gepumpt werden. Aufgrund seiner geringen Speicherkapazität für Sauerstoff und Energie bewirken bereits kurzfristige Ausfälle der Blutversorgung oftmals irreparable Hirnschäden. Die Länge aller Nervenbahnen des Gehirns eines erwachsenen Menschen beträgt etwa 5,8 Mio. Kilometer, das entspricht etwa dem 145-fachen Umfang der Erde (Wikipedia 2008a).

Die Verarbeitung von Information geschieht auf molekularer Ebene, beginnend mit der Erfassung membrangebundener Potenzialdifferenzen, deren physiologischer Umsetzung, bis hin zur (z.B. neuromuskulären) Aktivität des Gesamtorganismus. Mag die Aufklärung molekularbiologischer und biochemischer Mechanismen und ihrer Details im Einzelnen noch das tägliche Rüstzeug des physiologisch tätigen Neurologen sein, so sind größere Komplexe der Hirnforschung, insbesondere in der Bewusstmachung spezifischer Gehirnprozesse, aktueller Forschungsgegenstand. So treten grundlegende und zielgerichtete Fragen elektrochemischer Wechselwirkungen sowie Fragen echter bewusster 
Steuerung ganzer Gehirnzentren in den Vordergrund moderner Forschungsstrategien.

\section{Computer, künstliche Intelligenz und Internet}

Oftmals wird die Leistungsfähigkeit des menschlichen Gehirns mit dem Leistungsvermögen moderner Computersysteme verglichen. So schafft das menschliche Gehirn etwa $10^{13}$ bis $10^{16}$ analoge Rechenleistungen pro Sekunde, der IBM Computer Blue Gene/L bis zu 4,78 $\times 10^{14}$ Operationen pro Sekunde mit doppelter Genauigkeit. Hierbei muss berücksichtigt werden, dass die hohe Rechenleistung des Gehirns vor allem durch seine vielen parallelen Verbindungen und nicht durch eine hohe Geschwindigkeit bei den einzelnen Rechenvorgängen (Taktfrequenz) erzielt werden. Zusätzlich zur Parallelisierung stellt ein neuronales (biologisches) Netzwerk gleichzeitig sowohl Speicher- als auch Verarbeitungslogik dar, während diese bei Computern getrennt sind. Dies bedeutet, dass mit jedem Taktzyklus in einem neuronalem Netzwerk der gesamte Speicher aktualisiert wird, während ein Computer den Inhalt des Speichers schrittweise aktualisieren muss (Wikipedia 2008a).

Als Teilgebiet der Informatik entwickelte sich die Arbeitsrichtung der künstlichen Intelligenz (KI), die mit der Automatisierung intelligenten Verhaltens befasst ist. Hierbei versucht die starke KI, den Ersatz bzw. die Nachahmung des Menschen durch die Maschine und somit eine Intelligenz zu schaffen, die wie der Mensch denkt und Probleme löst und sich durch eine Form von Bewusstsein bzw. Selbstbewusstsein sowie durch Emotionen auszeichnet. Trotz unzähliger Bemühungen scheint eine Erfolg versprechende Forschungsstrategie derzeit nicht gefunden zu sein. Im Gegensatz hierzu versucht die sogenannte schwache KI die Simulation intelligenten Verhaltens mit Mitteln der Mathematik und der Informatik. Allerdings steht hier nicht die Schaffung von Bewusstsein oder ein tieferes Verständnis von Intelligenz im Vordergrund. Derzeit werden im Wesentlichen vier Intelligenzarten unterschieden: die visuelle, die sprachliche, die rationelle und die emotionale (Wikipedia 2008b).

Computer, die in diesen Bereichen eingesetzt werden, heißen Expertensysteme. Diese Expertensysteme basieren auf einer Datenbank, in der Fachwissen gespeichert ist. Darauf basierend kann das System, zusammen mit dem Anwender, Fachaufgaben lösen. Der Grundgedanke ist es, zu untersuchen, unter welchen Bedingungen Computer Verhaltensweisen von Lebewesen, die auf Intelligenz beruhen, nachvollziehen können.

Das Internet ist ein elektronischer Verbund von Rechnernetzwerken, mit dem Ziel, Verbindungen zwischen einzelnen Computern herzustellen und so Daten auszutauschen. Um spezifische Informationen zu finden, bedient man sich sogenannter Suchmaschinen. Die derzeit gängigste Such- maschine ist Google. Bei Suchmaschinenabfragen im deutschen Internet besitzt Google bereits einen Marktanteil von $83-90 \%$. Google bietet dabei wichtige Informationsquellen als Textdokumente, Bilddateien oder auch Videos an. Außerdem bietet Google diverse Dienste wie z. B. Gratis E-MailAdressen oder Übersetzungen von Webseiten in verschiedene Sprachen an. Allerdings nimmt sich Google auch das Recht, sämtliche E-Mail Nachrichten zu lesen und nach bestimmten Schlagworten zu durchsuchen, um dann diese Informationen als gezielte Werbemaßnahmen zu benutzen. So werden beispielsweise unsere persönlich geschriebenen E-Mails nach dem Wort Auto durchsucht und dann erhält man plötzlich aus allen Richtungen PKW-Werbung im Postfach.

Eine seit Jahren geübte Kritik an Google betrifft insbesondere die Vormachtstellung von Google hinsichtlich des Datenschutzes aber auch des Urheberrechts (Bergere und Osmont 2007).

So hatte Google im April 2007 für 3,1 Mrd. Dollar den Onlinewerbedienst DoubleClick gekauft und damit die Besorgnis vor einer Monopolstellung bei der Konkurrenz geweckt, da Google bei Textwerbung in Suchergebnissen bereits Marktführer ist. Durch den Kauf von DoubleClick (DoubleClick) eröffnete sich für Google auch eine gezielte Führungsposition bei Onlinewerbung mit Bildern und Videos. Neben den Wettbewerbern auf dem Markt haben sich auch Bürgerrechtsgruppen bei der Federal Trade Commission (US-amerikanische Wettbewerbsbehörde) für ein Verbot der Übernahme stark gemacht. Verbunden hiermit sind Bedenken im datenschutzrechtlichen Bereich, da sowohl DoubleClick als auch Google erhebliche Daten über das Surfverhalten von Internetnutzern generieren. Hieraus lassen sich für den Anbieter ganz gezielt persönliche Werbemaßnahmen für den Nutzer ableiten. Außerdem besteht die Gefahr, dass die erzeugten Daten weitergegeben und möglicherweise missbraucht werden (Heise 2007a,b). Microsoft möchte daher durch eine geplante (feindliche) Übernahme von Yahoo (www.yahoo.com) für fast 45 Mrd. US-Dollar aufschließen (u. a. Spiegel 2008).

\section{Emotionale Intelligenz}

Die in Kap. 2 aufgeführten Intelligenzen beziehen sich auf das in der Großhirnrinde lokalisierte kognitive Denken, das im Wesentlichen das logische Denken und Handeln unterstützt.

Bereits Ende der 1960er-Jahre entwickelte Joseph Weizenbaum vom MIT das Programm ELIZA, in dem der Dia$\log$ eines Psychiaters mit Patienten simuliert wird. Mit diesem Programm war es möglich, den Patienten die Illusion eines beseelten Gesprächspartners zu vermitteln. Weizenbaum selbst war entsetzt über die Wirkung des Programms auf Menschen und entwickelte sich vom Pionier der künst- 
lichen Intelligenz zum Computer- und Medienkritiker (Weizenbaum 1978). Unter http://www.tieben.de können Sie mit Eliza „plaudern“.

Daneben hat sich in den vergangenen Jahren die wissenschaftliche Untersuchung einer zweiten Gehirnstruktur entwickelt, die nach Goleman (1997) als emotionale Intelligenz bezeichnet wird. Ihr morphologischer Sitz als Informationsverarbeitungszentrum befindet sich ganz im Innern der Schädeldecke. Während das kognitive Gehirn - lokalisiert in der gefalteten Oberfläche des Neokortex - Wahrnehmung, Sprache und Denken fördert, übt das emotionale Gehirn eine Kontrollfunktion unserer eigenen Gefühlswelt aus. Die beiden Gehirne, das emotionale und das kognitive, nehmen die von der Außenwelt kommenden Informationen nahezu gleichzeitig auf. Danach können sie entweder gut zusammenarbeiten oder aber einander die Kontrolle über Denken, Gefühle und Verhalten streitig machen (Servan-Schreiber 2006; Schmid 2008). Das Resultat dieser Interaktion - Kooperation oder Konkurrenz - bestimmt unser Fühlen und unser Verhältnis zur Welt und zu anderen Menschen. Stehen die beiden Gehirne in Rivalität zueinander, fühlen wir uns schlecht bzw. unglücklich. Ergänzen sich hingegen emotionales und kognitives Gehirn und gibt das eine, und zwar das Emotionale, die Richtung vor, wie wir unser Leben gestalten wollen, während das andere, das Kognitive, uns dazu bringt, so klug wie möglich in eben dieser Richtung vorwärts zu gehen, verspüren wir eine innere Harmonie, die jeglichem dauerhaften Wohlbefinden zu Grunde liegt (Servan-Schreiber 2006). Das Gehirn ist dabei nicht so starr organisiert, dass diese jeweilige emotionale Verfassung als Kooperation bzw. Widerstreit unterschiedlicher Gehirnteile mit bildgebenden Verfahren optisch fassbar würde. Dessen ungeachtet gelingt es in Einzelfällen, etwa die in der Meditation erlangte besondere Harmonie oder aber schwere Depressionen und andere psychische Störungen durch derartige Methoden bildlich als Aktivitätsmuster bzw. -schwäche sichtbar zu machen.

Neben einer verstärkten psychologischen Beeinflussung medizinischer Gesundungsprozesse haben diese Arbeitsrichtungen auch starken Einfluss auf Kognitionspsychologen (Gigerenzer 2007), z. B. bei der Wirkungsforschung medialer Einflüsse auf den Menschen (Kast 2007). Depressionen, Zukunftsangst und Stress, also die modernen psychologischen „Volkskrankheiten“ unserer Zeit, sind unter Berücksichtigung dieser relativ neuen Emotionsmedizin teilweise ohne Medikamente und jahrelange Psychotherapie behandelbar (Servan-Schreiber 2006), da das emotionale Gehirn zahlreiche Körperfunktionen wie Herzrhythmus, Blutdruck, Hormonhaushalt, Verdauungs- und Immunsystem kontrolliert.

Eine ganz besondere Arbeitsrichtung beschäftigt sich mit der Untersuchung von Inselbegabten, auch Savants genannt. Dies ist ein Phänomen (Syndrom), bei dem Menschen, oft mit kognitiver Behinderung, in einem kleinen Teilbereich außergewöhnliche Leistungen vollbringen. $50 \%$ der bekannten Inselbegabten sind Autisten. Bereits der Drehbuchautor Barry Morrow erhielt durch einen Savant seine Inspiration für den Kinofilm „Rainman“. Savants fehlen häufig ausgeprägte Gefühlsausprägungen, sind aber oft in der Lage, phänomenale Gedächtnisdaten abzurufen, etwa Geschichts- oder Kalenderdaten oder aber eine erstaunliche Kreativität im Erzeugen vormals nie gedachter Gedanken (Einstein-Effekt) zu entwickeln.

\section{Ausblick}

Um auf dem Weg eines ökonomisch sinnigen, ökologisch verantwortbaren und multikulturell friedlichen internationalen Informationsprozesses auch künftig weiterzukommen, wird ein Gesamtkonzept benötigt. Dieses muss sowohl theoretisch grundlegend als auch wirtschaftlich handlungsfähig bzw. handlungsleitend sein, um durch entsprechende Information und Informationsverarbeitung effektiv handeln und gesundend wirken zu können.

Derzeit müssen wir teilweise feststellen, dass richtige und für einen Großteil der Menschheit zur Verfügung stehende Informationen aus Wirtschaft, Umwelt und Sozialem zum Teil unzureichend dargestellt werden bzw. nicht in ausreichendem Maße integrativ reflektiert sind (Markert et al. 2005, 2006; Tiessen et al. 2007).

Bürger der VR China - rund 20\% der Menschheit - haben überhaupt keinen unzensierten Zugang zum Internet, weitere Milliarden von Menschen können ihn sich nicht leisten. Sollte es vielleicht einmal zum „Krieg um Bildung“ (Laptop.org 2008) kommen?

Hinzu kommt die sich in den vergangenen Jahren in Deutschland (und Europa) immer weiter zuspitzende Situation, dass Nöte und Bedürfnisse nicht gesamtbefriedigend gelöst werden können. Hieraus ergibt sich die Forderung eines ganzheitlichen Informations- und Lösungskonzeptes unter Berücksichtigung spezifischer kulturhistorischer und individuell gelagerter Bedürfnisse. Insbesondere müssen kognitive und emotionale Informationsprozesse gleichbedeutend in die Intelligenzforschung einfließen.

Den Versuch, naturwissenschaftliche Kenngrößen und multikulturelle Kernsätze über eine Kalibriergerade „dialogische Bildung" auch in einem zeitlichen Rahmen wiederzugeben, zeigt Abb. 1.

Hierbei ist es wichtig, für Menschen unterschiedlichster Herkunft ein Wertesystem und Bildungssystem zu erarbeiten, das individuelle Bedürfnisse und gemeinschaftliche Verpflichtungen gleichermaßen berücksichtigt. Denn Intelligenz scheint die besondere Fähigkeit zu sein, Beziehungen und Zusammenhänge zwischen einzelnen Informationsquellen herzustellen. Dabei stehen Liebe und Macht seit Menschengedenken in Konkurrenz. Liebe (zu sich selbst, zu 


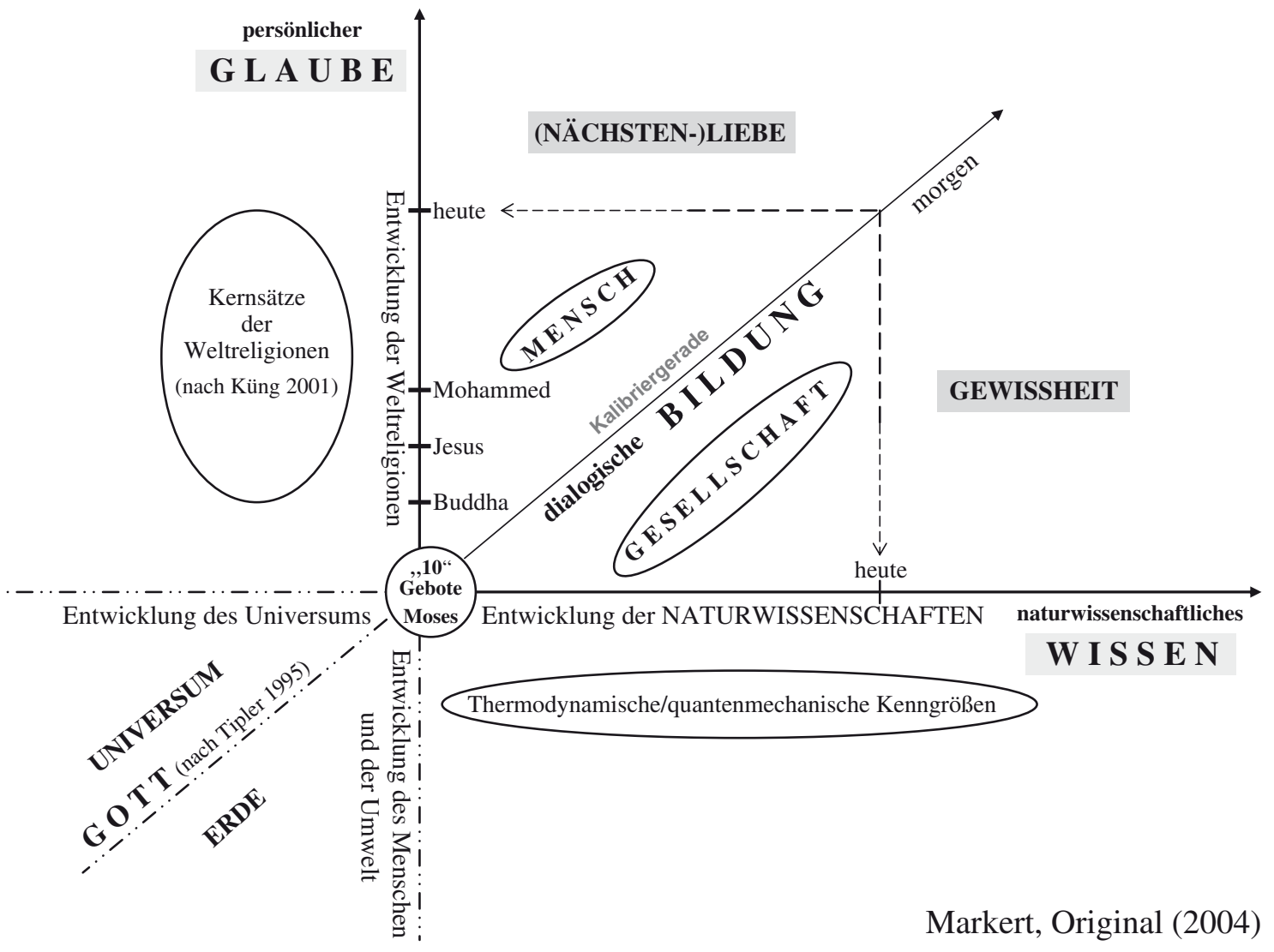

Abb. 1 Informationserzeugung als „De Novo Synthese“ aus naturwissenschaftlichem Wissen, weltreligiösem Glauben und dialogischer Bildung (aus Markert et al. 2005)

anderen und zur Natur) fördert positive Beziehungen, Macht verleitet sehr oft zum Missbrauch und daher zur Förderung negativer Beziehungen (Janisch 2007).

\section{Literatur}

Bergere S, Osmont S (2007) Wer hat Angst vor Google? Ein Dokumentarfilm vom 20. April 2007, ARTE

DoubleClick http://www.doubleclick.com

Gigerenzer G (2007) Bauchentscheidungen, die Intelligenz des Unbewussten und die Macht der Intuition. Bertelsmann, München

Goleman D (1997) Emotionale Intelligenz. DTV, München

Heise (2007a) http://www.heise.de/newsticker/meldung/88379

Heise (2007b) http://www.heise.de/newsticker/meldung/90306

Janisch W (2007) Was Terroristen für die Wahrheit halten. Meppener Tagespost, 24. April, S 4

Kast B (2007) Wie der Bauch beim Denken hilft. Die Kraft der Intuition. Fischer Verlag, Frankfurt

Laptop.org (2008) http://laptop.org/index.de.html

Markert B, Fränzle S (2007) Information und Informationsverarbeitung: Die Stufen der Erkenntnis und das Verhältnis von Objektivität und Subjektivität. Umweltwiss Schadst Forsch 19(2):86-90
Markert B, Fränzle S, Hosang M (2005) Vorzeichenwechsel - Wie Gesellschaft sich verändern kann. Peter Lang, Europäischer Verlag der Wissenschaften, Frankfurt am Main

Markert B, Lieth H, Menke-Glückert P, Hosang M, Fränzle S (2006) Zur Existenz eines ganz starken anthropischen Prinzips: Ist Gott ein Perpetuum mobile nullter Art? BOD-Verlag, Norderstedt

Radio Bremen (2006) Expedition ins Gehirn - 3-teilige WissenschaftsDokumentation. Erstausstrahlung: 20.-22. Februar 2006

Roth G (2003) Aus Sicht des Gehirns. Suhrkamp Verlag, Frankfurt am Main

Schmid W (2008) Glück. Insel Verlag, Frankfurt am Main

Servan-Schreiber D (2006) Die Neue Medizin der Emotionen, 5. Aufl. Wilhelm Goldmann Verlag, München

Spiegel (2008) www.spiegel.de/wirtschaft/0,1518,532565,00.html

Spitzer M (2007) Geist und Gehirn. Sendereihe in BR-alpha, November/Dezember 2007

Tiessen H, Brklacich M, Breulmann G, Menenzes R (Hrsg) (2007) Communicating global change science to society: an assessment and case studies. SCOPE 68, Island press, London

Weizenbaum J (1978) Die Macht der Computer und die Ohnmacht der Vernunft. Suhrkamp Verlag, Frankfurt am Main

Wikipedia (2008a) de.wikipedia.org/wiki/Gehirn

Wikipedia (2008b) de.wikipedia.org/wiki/künstliche_Intelligenz 